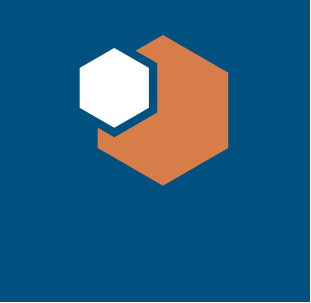

\section{Materials advances result from study of cold fusion}

\section{By Philip Ball}

$\mathrm{I}_{\mathrm{a}}^{\mathrm{n}}$ 1989, electrochemists Stanley Pons and Martin Fleischmann, working at The University of Utah, astounded the scientific world by announcing that they had achieved nuclear fusion of hydrogen isotopes - the process that powers stars - in a simple benchtop experiment. They claimed to have seen evidence of fusion, in particular a heat output greater than the energy input to the apparatus, when they electrolyzed deuterated lithium hydroxide in heavy water using palladium electrodes.

The claim created excitement, controversy, and even outrage. For one thing, the "breakthrough" was announced not in a scientific paper but in a press conference - then still a highly irregular departure from scientific protocol. Pons and Fleischmann released only the sketchiest details of their experimental procedure and results for what became known as "cold fusion," and even these details seemed to change as the claims were probed and challenged. Some other researchers reported similar findings, but several large-scale efforts to replicate the Utah work failed to see "excess heat" or any other evidence of fusion. The work came to be widely regarded as a textbook case of "pathological," irreproducible science; some even suspected fraud.

All the same, cold fusion has never gone away. A few researchers, working at the fringes of the scientific community, have continued to claim to see tantalizing signs that there really is something in it after all. However, the field has never shaken off its bad reputation. There was much surprise when in June, 30 years after the original event, Nature published an article by a team of researchers funded by Google describing renewed searches for "low-energy" fusion of hydrogen isotopes (deuterium, which has a lower energy threshold for fusion than hydrogen-1) using palladium electrodes. ${ }^{1}$

The paper reported no evidence of such a process in electrochemical experiments similar to those of Pons and Fleischmann, but it described a low level of fusion from a different experimental setup in which a plasma of deuterium ions surrounded a negatively charged palladium wire. The new findings will not persuade anyone that Pons and Fleischmann were right, but they could give cold fusion a new lease on life. Moreover, the study showed that there are interesting things still to learn about the materials science of the palladium-hydrogen system.

That is what attracted materials researcher Yet-Ming Chiang of the Massachusetts Institute of Technology (MIT) in Cambridge, Mass., to the collaboration. Chiang says that he and the other research team members were recruited by Matt Trevithick, a former MIT graduate and now a program manager at Google Research in Mountain View, Calif., who is a co-author of the article. Trevithick had maintained an interest in cold fusion ever since the story broke. Chiang, in contrast, says that he paid it rather little heed at the time, when he had only recently become a faculty member at MIT. At that stage, Chiang was busy with hightemperature superconductivity, being on one of the teams that formed the spinout company American Superconductor.

When Trevithick contacted him to ask if he would be interested in looking into cold fusion, Chiang had no strong preconceptions. Their co-author, electrochemist Curtis Berlinguette of The University of British Columbia in Vancouver, Canada, meanwhile, was genuinely enthusiastic about the prospect.

"Renewable energy and fusion technologies are not scaling at the pace we need them to," says Berlinguette. "If cold fusion were realizable, it could take the world into an era of energy surplus rather than scarcity. It therefore seemed irresponsible to not take another look at it. For me, cold fusion started in 2015," says Berlinguette. "Prior to that, I didn't know enough about it to have an opinion. I was driven simply by curiosity to learn more about the field."

Although the research team began by consulting leading figures in the cold-fusion community and designing replication studies, "we quickly started designing entirely new experiments to better inform the materials and physics space relevant to cold-fusion research," says Berlinguette. "Our overarching objective was to conduct hypothesis-driven research that could be published in top science journals."

For Chiang, there was the possibility of delving into some interesting materials science - in particular, the remarkable capacity of palladium to absorb hydrogen. "Palladium is not the only material that will absorb hydrogen at high concentrations, but it is the exemplar," says Chiang. He saw a clear connection to his work on ion-insertion compounds for batteries. Electrochemistry, he says, is known to be an extremely powerful driving force for loading ions or atoms into such materials. Could they harness that force to get higher concentrations of hydrogen into the metal?

That alone would not guarantee fusion, though. "There were several hypotheses mixed up in the original proposal," says electrochemist David Williams of The University of Auckland in New Zealand, who led a major British attempt to replicate the claims of Pons and Fleischmann in 1989. "First there was the idea that loading hydrogen into palladium would obtain a sufficient density to trigger fusion reactions," which he says was based on flawed assumptions. "Hidden inside this hypothesis was an assumption 
that the absorbed hydrogen had sufficient kinetic energy to induce fusion collisions. Alternatively, there is the hidden hypothesis that the electrons associated with the heavy metal palladium somehow screen the repulsion between hydrogen atoms."

Finally, some supposed that "there would be something special about fusion in a solid matrix that was different to fusion caused by collisions in a high-temperature plasma." Thus, says Williams, "the original cold-fusion idea was in fact a Russian doll of hypotheses, each of which had limited plausibility."

Chiang says there has been a feeling among those still researching cold fusion that, to have any chance of success, researchers need to get to $\mathrm{H}: \mathrm{Pd}$ ratios approaching 1:1. "Palladium hydride is actually a very simple material from a crystal-chemical point of view," he says. The metal itself has a face-centered-cubic structure, and hydrogen atoms occupy the octahedrally coordinated interstitial sites between palladium atoms. If these are fully occupied, the resulting hydride has a rock-salt (sodium chloride) structure. But it is difficult to get to that ratio under ambient conditions, says Chiang. The hydrogen loading tails off at a ratio of about
0.7 , and to get it any higher, the hydrogen pressure needed increases exponentially.

Chiang says that much of his effort in the project focused on making materials for experiments conducted by physicist Thomas Schenkel's group at Lawrence Berkeley National Laboratory in California, which took a completely different approach from the electrolysis studies of Pons and Fleischmann. Schenkel's group creates a plasma of deuterium ions in a vacuum chamber using high-voltage pulses applied to $\mathrm{D}_{2}$ gas, and then accelerates them toward a negatively charged metal (palladium) wire so that they bombard the metal. ${ }^{1,2}$ Some of the ions are incorporated into the palladium, where the deuterium nuclei can collide with one another and fuse to produce helium. This is somewhat like fusion created in particle accelerators, albeit at lower and less welldefined energies. Schenkel's studies have confirmed that D-D fusion can occur this way, as shown by the production of various fusion products: neutrons, helium-3, and tritium nuclei, for example.

The idea behind the technique is that "screening" of the positive charge of the deuterium nuclei by the electrons in the metal lattice can reduce the Coulomb

repulsion barrier to fusion of two nuclei. The researchers observed rates of fusion, as measured by the neutron yield, approximately 100 times greater than what theory predicts at these energies - and it is not yet clear why. "I'd like to learn what the microscopic mechanisms are," says Schenkel, adding that "Our understanding of electron screening processes is apparently lacking." All the same, Chiang is careful to point out that the discrepancy is "the difference between almost nothing and slightly greater than nothing."

The researchers also conducted electrochemical experiments more like those of Pons and Fleischmann, using electric fields to absorb hydrogen from an electrolyte into a palladium cathode and measuring the heat output using calorimetry. They saw no sign of the "excess heat" that the Utah duo reported 30 years ago. Chiang stresses that to his knowledge, no experiment has unambiguously done so, once all energy sources and sinks are fully accounted for. However, "one of the things we learned a great deal about is how difficult it is to measure heat very precisely," he says.

As well as using an aqueous electrolyte, the group used other protonconducting electrolytes as well, including Nafion (a soft polymer) and the high-temperature ceramic barium cerium yt-

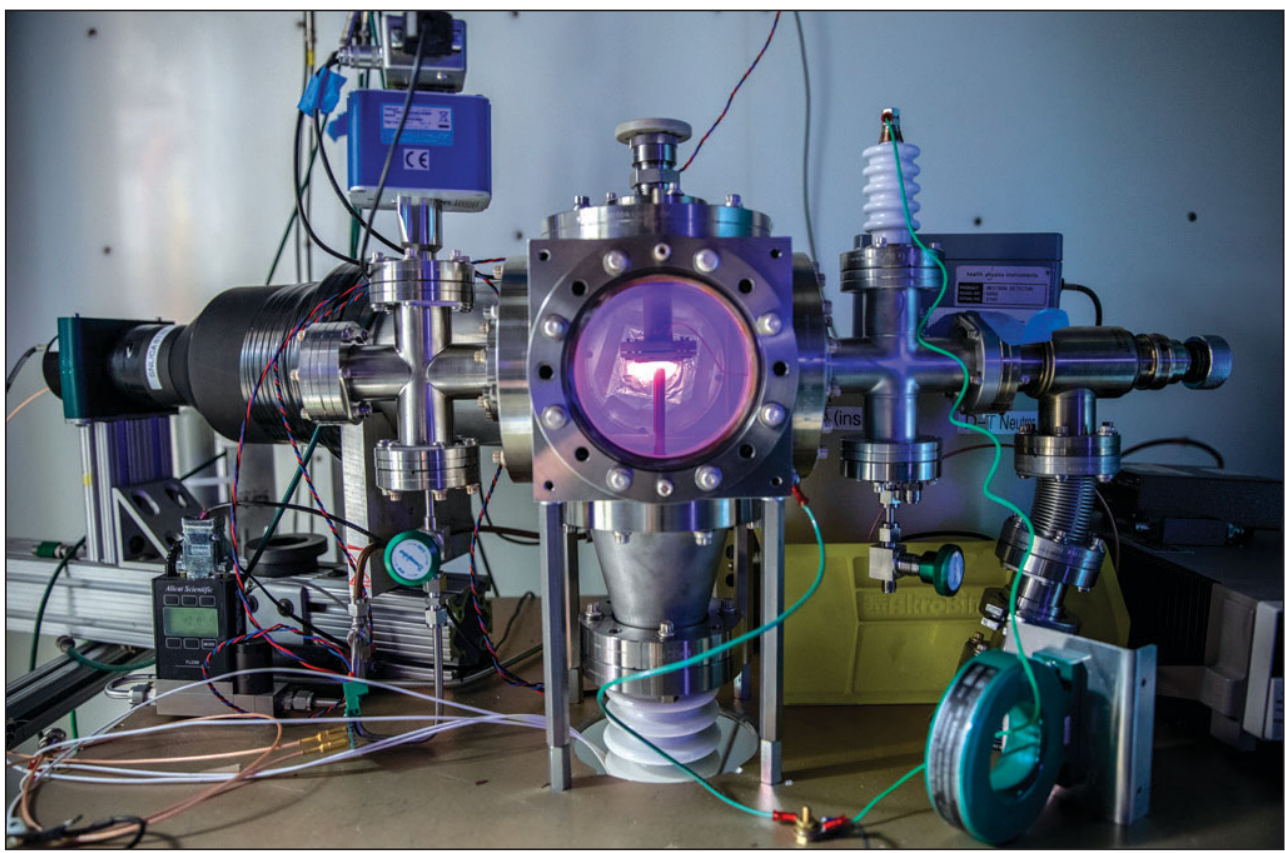
trate. They found that the amount of hydrogen loading depends critically on how intimately the electrolyte contacts the electrodes. As the metal takes up hydrogen, the lattice swells and the material can crack and crumble. "The electrochemically driven volume changes are critical to the outcome," says Chiang. "When the electrolyte is conformal, we're able to load to much higher concentrations. It's really a mechanical failure problem that limits what you can do." They could get a loading ratio of 0.9 with the Nafion electrolyte, which is soft enough to adapt to changes in the electrode. Since such 
"dry" experimental configurations make some types of measurement easier, they determined that using the polymer for loading is the best approach.

Loading the metal with hydrogen is like filling a leaky bucket: hydrogen gas is evolved even as it is absorbed. The relative rates of these two processes establish the steady-state loading level. However, researchers would like to see if they could get to much higher loading by forcing the hydrogen atoms into different sites in the metal lattice: those with tetrahedral rather than octahedral coordination. In theory that could give a ratio of up to $2: 1$ - and furthermore, the sites are closer together, which could possibly make fusion more likely. Chiang and his colleagues are now collaborating with others to use density functional theory to calculate the stability of the various hydride phases.

The response to the Nature report has been interesting, says Chiang. "We have received almost no negative responses, but a lot of positive, encouraging ones." Curiously, some of the reservations were from the cold-fusion community, who felt that publishing negative results might hurt the field. "This project was perceived to be risky for all stakeholders-PIs [principal investigators], students and postdocs, universities, sponsors - and so we are grateful that everyone has responded with an open mind about our efforts," says Berlinguette.

However, some researchers may wonder if the cold-fusion label is much more than canny advertising. "If materials science wishes to put funding into studying the Pd-H system, that makes sense," says particle physicist Frank Close, an emeritus professor at the University of Oxford who wrote a book on the original cold-fusion episode. "But if that was the name of the game, it wouldn't be making any profile outside a technical community."

Close wonders if the association with cold fusion might backfire, tainting what is otherwise serious research. "The moniker 'cold fusion' has become attached to a whole raft of things," says Close. "At one end of the scale, it is snake-oil people using a label to get funding from naïve people. At the other end of the scale, serious endeavors to study complicated
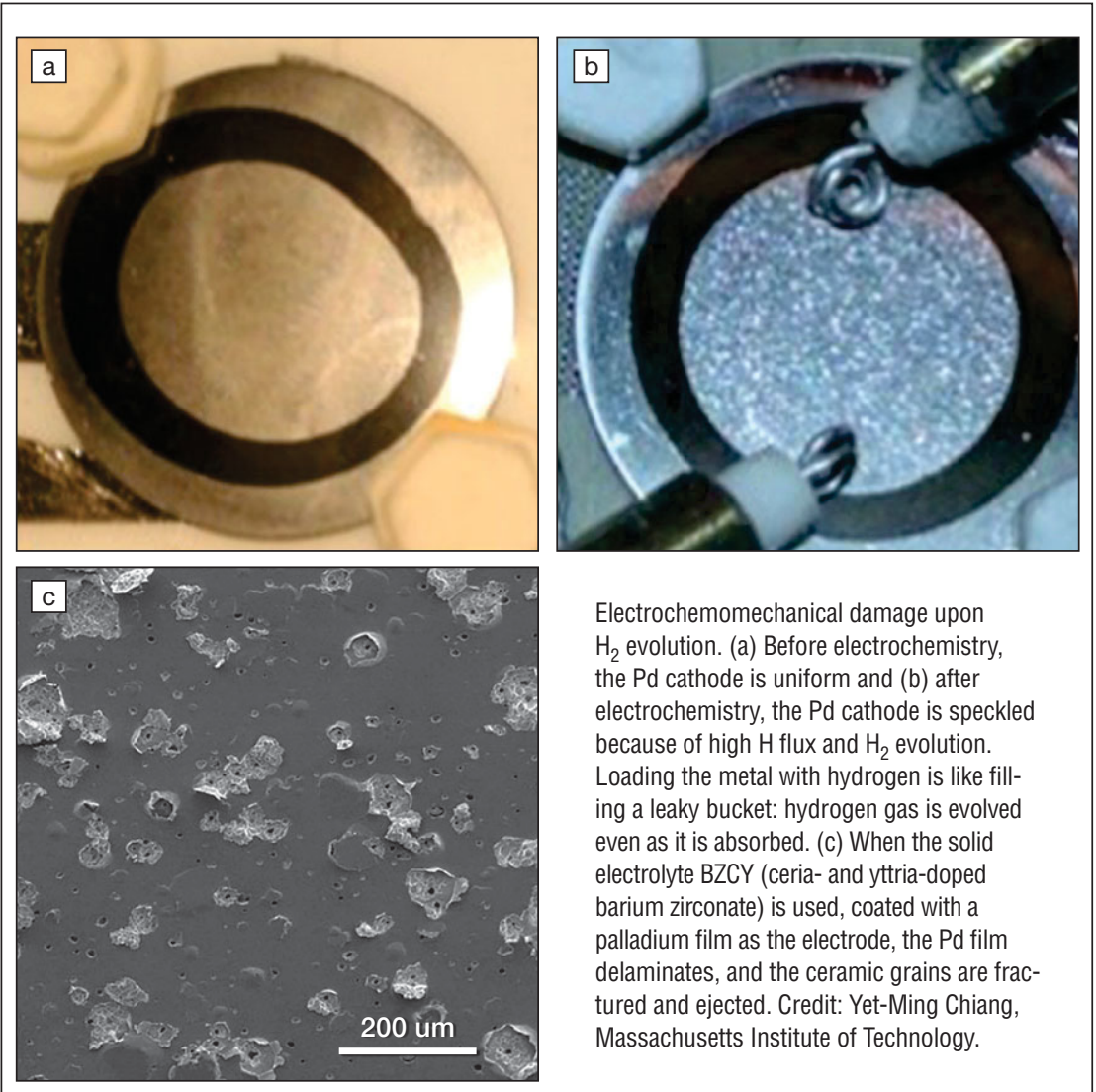

Electrochemomechanical damage upon $\mathrm{H}_{2}$ evolution. (a) Before electrochemistry, the Pd cathode is uniform and (b) after electrochemistry, the Pd cathode is speckled because of high $\mathrm{H}$ flux and $\mathrm{H}_{2}$ evolution. Loading the metal with hydrogen is like filling a leaky bucket: hydrogen gas is evolved even as it is absorbed. (c) When the solid electrolyte BZCY (ceria- and yttria-doped barium zirconate) is used, coated with a palladium film as the electrode, the Pd film delaminates, and the ceramic grains are fractured and ejected. Credit: Yet-Ming Chiang, Massachusetts Institute of Technology.

science of materials can be compromised by having the label attached to them."

At any rate, Close doubts whether more experiments looking for cold fusion will settle the matter. "In science, one cannot prove a negative," he says. "Do enough trials, with enough variables, and by random chance, there will be one or two or more that appear to be showing signals. That is the bane of the particle physicist's life!"

However, Williams feels that the authors of the new study "have really pushed the envelope of measurement science, which in itself is an important contribution." Of course, he adds, with speculative work like this, "you might find nothing along the lines of your original motivation, but you will surely find something, and who knows whether that will be important or not?"

While the work never seemed likely to make much impact on energy production, says Williams, "hydrides are interesting in their own right in relation to energy problems." As well as hydrogen storage, he points to a complex hydride that is a lithium-ion superionic conductor, ${ }^{3}$ and to hydride-ion conductivity in oxygen-doped lanthanum hydride ${ }^{4}$ and in $\mathrm{BaH}_{2}$ at high temperature. ${ }^{5}$

One person who found the work stimulating was Russell Hemley, a high-pressure materials chemist at the University of Illinois at Chicago. He suspects that the hydrogen loading of palladium might be increased by pressure. Hemley and his co-workers have already seen the 1:1 stoichiometric hydride for both hydrogen and deuterium at pressures of up to $100 \mathrm{GPa},{ }^{6}$ but have so far been unable to produce any more extensively hydrided stoichiometric phases. Hemley speculates that electrochemistry could augment the effects of high pressure in efforts to attain them.

What interests him in particular is not fusion but the possibility that this system might show superconductivity at relatively high temperatures, by analogy with such behavior that Hemley and his co-workers recently reported for lanthanum superhydride $\left(\mathrm{LaH}_{10}\right)$ at $260 \mathrm{~K}$ and $200 \mathrm{GPa}^{7}$ " "We're continuing to look into this," he says. "I have an open mind and hope some key experiments will lead to definitive results." 
"If you do experiments carefully and describe them openly, the results, whatever they are, don't provoke the kinds of negative responses they might otherwise," says Chiang. But having Google as a sponsor was important, he adds. "Being such a controversial project, I think it would have been very difficult to undergo a typical research proposal peer-review process and arrive at a point where a government agency would be willing to fund it." One of the objectives was to "see if we could re-open interest in research in this area in such a way that it would be legitimized."

Schenkel thinks that for his plasma experiments, a key question now is whether "we can control materials such as metal hydrides so that fusion yields increase further and at lower energies." He admits that he simply does not know if it will be possible, "but we have a list of experiments that can test well-defined hypotheses."

Besides, the new studies have spun off in entirely different directions. The ability of palladium to accommodate large concentrations of hydrogen could have implications in catalysis and hydrogen storage, says Chiang. He adds that the dramatic volume changes accompanying hydrogen loading could give the palladium hydride system the potential to act as an electrochemically switchable mechanical actuator.

And from out of the Google-funded work, Berlinguette's graduate student Rebecca Sherbo developed a palladiummembrane reactor that uses electricity to drive efficient hydrogenation (or deuteration) chemistry of small molecules. ${ }^{8}$ "This work was made possible because of the understanding [Sherbo] gained of the hydrogen diffusion properties in palladium electrodes," says Berlinguette. This work "has already prompted a number of leading research groups to start using it," he says, and it might find applications in the pharmaceutical industry for deuterating drugs. "We went looking for cold fusion, and we are now affecting drug design," he says.

\section{References}

1. C.P. Berlinguette, Y.-M. Chiang, J.N. Munday, T. Schenkel, D.K. Fork, R. Koningstein, M.D. Trevithick, Nature 570, 45 (2019).

2. T. Schenkel, A. Persaud, H. Wang, P.A. Seidl, R. MacFadyen, C. Nelson, W.L. Waldron, J.-L. Vay, G. Deblonde, B. Wen, Y.-M. Chiang, B.P. MacLeod, Q. Li (2019), https://arxiv.org/abs/1905.03400.

3. S. Kim, H. Oguchi, N. Toyama, T. Sato, S. Takagi, T. Otomo, D. Arunkumar, N. Kuwata, J. Kawamura S. Orimo, Nat. Commun. 10, 1081 (2019).

4. K. Fukui, S. limura, T. Tada, S. Fujitsu, M. Sasase, H. Tamatsukuri, T. Honda, K. Ikeda, T. Otomo, H. Hosono, Nat. Commun. 10, 2578 (2019).

5. M.C. Verbraeken, C. Cheung, E. Suard, J.T.S. Irvine, Nat. Mater. 14, 95 (2015).

6. K. Brownsberger, M. Ahart, M. Somayazulu, C. Park, S.A. Gramsch, R.J. Hemley, J. Phys Chem. C 121 (49), 27327 (2019).

7. R.J. Hemley, M. Ahart, H. Liu, M. Somayazulu, Proc. Ramón Areces Symp. "Superconductivity and Pressure: On the Road to Room-Temperature Superconductivity" (Madrid, Spain, May 21-22, 2018).

8. R.S. Sherbo, A. Kurimoto, C.M. Brown, C.P. Berlinguette, J. Am. Chem. Soc. 141, 7815 (2019).

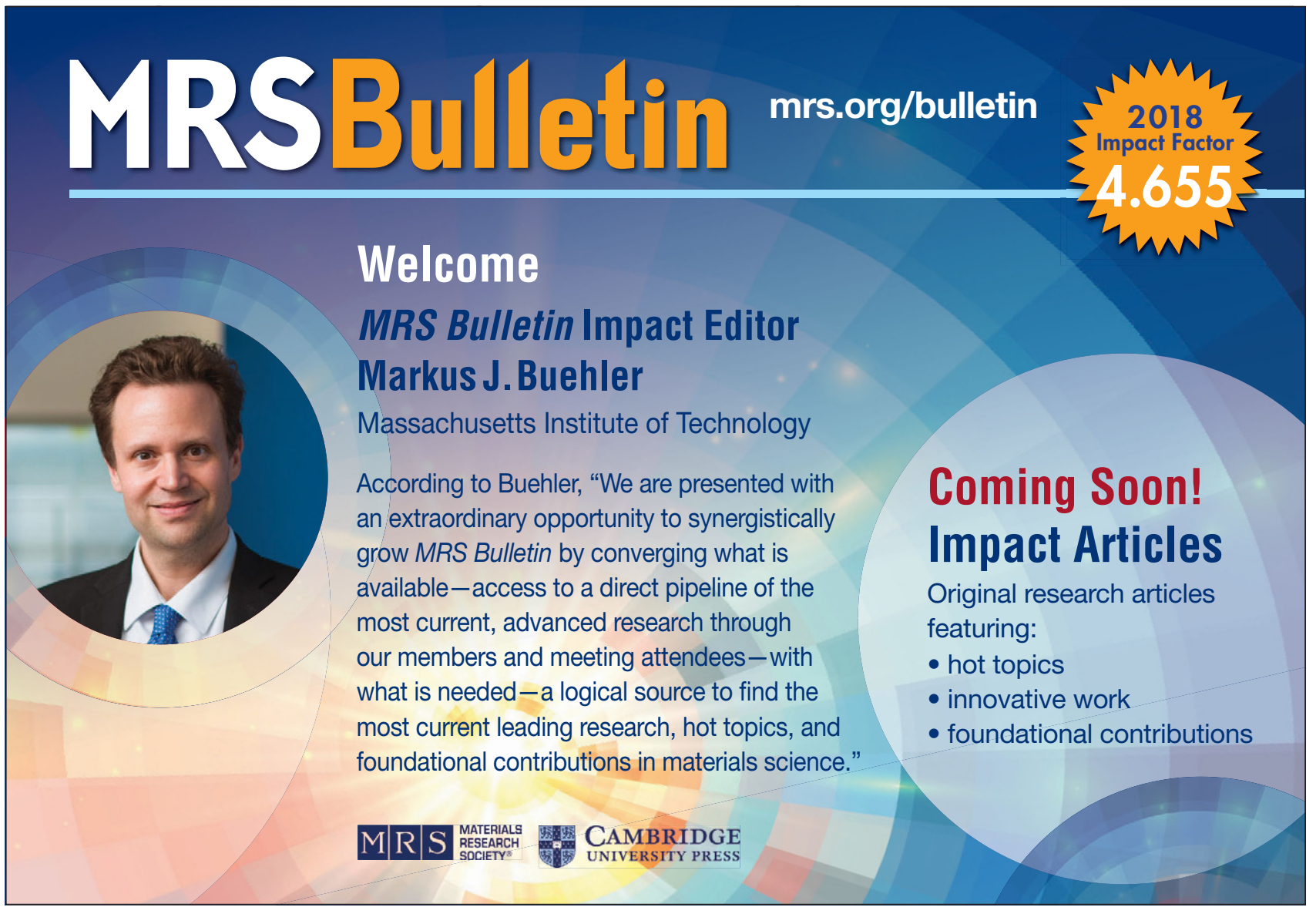

